\title{
Alternative anticoagulation therapies for antiphospholipid syndrome - a new therapeutic challenge
}

\author{
Diana Mazilu ${ }^{1,2}$, Daniela Opris-Belinski ${ }^{1,2}$ \\ 1"Carol Davila" University of Medicine, Bucharest, Romania \\ 2"Sfanta Maria" Clinical Hospital, Bucharest, Romania
}

\begin{abstract}
The major treatment issues in Antiphospholipid Syndrome (APS) include the the prevention of first thrombosis, the treatment of acute thromboembolic manifestations, the choice of anticoagulation, the duration of anticoagulation and secondary thrombosis prevention. Anticoagulation should individualized to the patient and clinical setting. Clasical anti vitamin $\mathrm{K}$ antagonists, warfarin and acenocumoral, carry some risks and disadvantages. Direct thrombin inhibitor and direct anti-Xa inhibitors are currently available and were approved for the prevention of stroke and systemic embolism in patients with atrial fibrillation, for the treatment of deep vein thrombosis (DVT) and for the prevention of recurrent DVT and pulmonary embolism. Besides many case series reports, there are ongoing clinical trials testing their efficiency and safety in patients with APS. In this paper we reviewed the advantages, risks and disadvantages for the use of direct oral anticoagulants in this category of patients.
\end{abstract}

Keywords: antiphospholipid syndrome, direct oral anticoagulants, advantages and risks

\section{INTRODUCTION}

Antiphospholipid syndrome (APS) is a systemic autoimmune disease characterized by venous or arterial thrombosis and/or pregnancy loss in the presence of persistent expression over time of antiphospholipid antibodies (aPL): lupus anticoagulant (LAC), anticoardiolipin (aCL) and $\beta 2$-glycoprotein (anti-ß2-GLI). APS can occur as a primary condition, or in the presence of a systemic autoimmune disease (1).

The serological profile that is associated with high risk of recurrent thrombosis in patients with APS consists of the presence of LAC, triple positivity (LAC, aCL and anti- $\beta 2-\mathrm{GLI}$ ) and the persistence positivity for aCL in medium-high titers. The presence of systemic lupus erythematosus (SLE), coexistent cardiovascular risk factors, personal history of arterial thrombosis and recurrent thrombosis despite anticoagulant therapy are clinical features that increase the risk for further recurrent thrombosis in patients with APS (1).
The major treatment issues in APS include the the prevention of first thrombosis among patients with aPL that do not meet criteria for APS, the treatment of acute thromboembolic manifestations, the choice of anticoagulation, the duration of anticoagulation and secondary thrombosis prevention.

\section{Issue for classic anticoagulation}

Oral anticoagulation is a provocative treatment. Warfarin and related vitamin $\mathrm{K}$ antagonists (VKAs) block the function of the vitamin $\mathrm{K}$ epoxide reductase complex in the liver, leading to depletion of the reduced form of vitamin $\mathrm{K}$ that serves as a cofactor for gamma carboxylation of vitamin K-dependent coagulation factors (2). The epoxide reductase is needed to recycle vitamin $\mathrm{K}$ between reduced and epoxide forms. Without gamma carboxylation, the vitamin K-dependent factors, including factors II (prothrombin), VII, IX, and X, are immunologically detectable, but they cannot function because they 
cannot adequately bind calcium and phospholipid membranes needed for their hemostatic function (3).

It is well known that APS patients may encounter challenges with monitoring the anticoagulation therapy due to the effects of the aPL on the activated partial thromboplastin time $(a P T T)$ and prothrombin time $(P T)$ /international normalized ratio $(I N R)$ assays. It is important to obtain a baseline PT/INR and aPTT before starting anticoagulation in APS so that a baseline prolonged PT or aPTT is not misinterpreted as an effect of an anticoagulant.

Factors that affect the dose-response relationship between VKAs dose and INR include the following: vitamin $\mathrm{K}$ intake, medication adherence, genetic variation, drug interactions, smoking and alcohol use, renal, hepatic and cardiac function and hypermetabolic states. High vitamin $\mathrm{K}$ foods are collard greens, kale, spinach, brussels sprouts. There is a long list of medications that may interfere with the effect of VKAs. Acetaminophen, allopurinol, amiodarone, antibiotics as penicillins, doxycycline, cephalosporins, fluoroquinolones, metronidazole are just some the medications that may increase INR. Azathioprine, sucralfate, and antiepileptic drugs may decrease INR. Patients with severe chronic kidney or liver disease are at increased risk for erratic INR results as well as for hemorrhagic complications. Closer attention to monitoring and dose adjustments is often indicated in these settings and, in some cases, the risks of anticoagulant therapy may outweigh the potential benefit.

Concurrent thrombocytopenia may increase concerns about bleeding. An anticoagulant may be administered as long as the platelet count exceeds 50,000 to $60,000 / \mathrm{microL}$ and is not declining. Of course, the risks and benefits of anticoagulation for each patient should be weighted. Studies show that thrombocytopenia does not reduce the risk of thrombosis in APS and should not be interpreted to have a protective effect (4).

Another important issue is the genetic polymorphism that has been implicated in altered sensitivity to vitamin $\mathrm{K}$ antagonists (VKAs). The genetic testing is not routinely recommended. The most important genes involved in VKA sensitivity are vitamin $\mathrm{K}$ epoxide reductase, subunit 1 (VKORC1), the drug target; and hepatic cytochrome P450 2C9 isoform (CYP2C9), which metabolizes the drug to an inactive form.

A number of polymorphisms in the gene encoding a subunit of the complex, VKORC1, have been identified and demonstrated to affect dose requirements of warfarin and other VKAs. VKORC1 haplotypes can be used to stratify patients into low-, intermediate-, and high-dose warfarin groups and may explain differences in dose requirements among patients of different ancestries. The molecular mechanism of this warfarin dose response appears to be regulated at the transcriptional level(5). Initial variability in the INR response to warfarin is strongly associated with genetic variability in the pharmacologic target of warfarin, VKORC1 (6).

The genetic variation in the CYP2C9 gene has been demonstrated to affect warfarin and acenocoumarol dose requirements, although to a lesser degree than VKORC1 variants in some studies. The simple genotyping of 2 single-nucleotide polymorphisms (SNPs), VKORC1 $-1639 \mathrm{G}>\mathrm{A}$ or $1173 \mathrm{C}>\mathrm{T}$ and the CYP2C9*3 polymorphisms, could thus predict a high risk of overdose before initiation of anticoagulation with acenocoumarol, and provide a safer and more individualized anticoagulant therapy (7).

Genetic variation in VKORC1 and CYP2C9 are not the only ones implicated in INR control.

Genetic screening using a panel of drug-metabolizing enzyme and drug transporter polymorphisms identified a variant in the cytochrome P450 4F2 isoform (CYP4F2) that correlated with differences in warfarin dose requirement. DNA variant (rs2108622; V433M) in cytochrome P450 4F2 (CYP4F2) was associated with a difference in warfarin dose of approximately $1 \mathrm{mg}$ /day between CC and TT subjects (8). However, this variant only accounts for approximately 2 percent of inter-patient variability; in contrast, VKORC1 and CYP2C9 genotype together account for 37 percent of variability. A role for the same $C Y P 4 F 2$ variant was also demonstrated for acenocoumarol in a cohort of 100 individuals, underlining the relevant role of CYP4F2 $\mathrm{V} 433 \mathrm{M}$ polymorphism in the pharmacogenetics of coumarin anticoagulant(9).

\section{Direct oral anticoagulants (DOAC) in patients with Antiphospholipid Syndrome}

Direct thrombin inhibitor (dabigatran etexilate) and direct anti-Xa inhibitors (rivaroxaban, apixaban and edoxaban), are currently available. Their efficacy has been demonstrated in large phase III clinical trials, and both rivaroxaban and dabigatran have been approved for the prevention of stroke and systemic embolism in patients with atrial fibrillation 
(AF). Rivaroxaban and apixaban were licensed for the treatment of deep vein thrombosis (DVT) and for the prevention of recurrent DVT and pulmonary embolism (PE) following acute DVT in adults based on the results of the EINSTEIN-DVT, EINSTEIN-PE study and AMPLIFY international multicentre randomized trials. The RE-COVER trial showed that for the treatment of acute VTE, a fixed dose of dabigatran was as effective as warfarin, had a safety profile that was similar to that of warfarin, and did not require laboratory monitoring (10-13). In randomized control trials (RCTs) assessing the therapeutic dose of DOACs $v s$ VKAs, warfarin at a target INR of 2.5 (i.e. range 2.0-3.0) has been used as the comparator (13)(11)(12). Regarding the existing data on DOACs efficiency in APS patients, there are a number of case series and case reports published (14).

\section{Rivaroxaban for Antiphospholipid Syndrome}

Rivaroxaban for Antiphospholipid Antibody Syndrome (RAPS), is a randomised, controlled, open-label, phase II-III, non-inferiority trial. This study enrolled patients with APS who were taking warfarin for previous venous thromboembolism, with a target international normalised ratio of 2,5. Patients were randomly assigned 1:1 to continue with warfarin or receive $20 \mathrm{mg}$ oral rivaroxaban daily. Randomisation was done centrally, stratified by centre and patient type (with $v s$ without systemic lupus erythematosus). The primary outcome was percentage change in endogenous thrombin potential (ETP) from randomisation to day 42, with non-inferiority set at less than $20 \%$ difference from warfarin in mean percentage change. Analysis was by modified intention to treat. Other thrombin generation parameters, thrombosis, and bleeding were also assessed. Treatment effect was measured as the ratio of rivaroxaban to warfarin for thrombin generation.

One hundred sixteen patients were included in this trial. When anticoagulation intensity was assessed by percentage change in ETP alone, rivaroxaban was inferior to warfarin in patients with APS and previous venous thromboembolism. However, peak thrombin generation was lower with rivaroxaban and, therefore, the overall thrombogram indicated no difference in thrombotic risk. Despite the fact that $28 \%$ of patients in RAPS had triple positivity for lupus anticoagulant and antibodies against cardiolipin and $\beta_{2}$ GPI at baseline and, therefore, had a particularly high-risk antibody profile, no new thrombotic events were seen during 6 months of treatment. No patients had major bleeds, and the frequencies of clinically relevant and minor bleeding were similar in the two groups (15). A limitation of RAPS is that it was not designed to confirm clinical efficacy and long-term safety. Rather, the trial was designed with a laboratory surrogate outcome measure to assess the mechanism of action of the interventions in patients with thrombotic APS with previous venous thrombembolism (15).

Although the findings of Cohen and colleagues are hopeful, the efficacy of rivaroxaban treatment for secondary prevention of thrombosis in patients with APS remains unclear. The outcomes of larger clinical trials investigating these questions are awaited.

Another important clinical trial is Rivaroxaban in Thrombotic Antiphospholipid Syndrome (TRAPS). It is a prospective, randomized clinical trial comparing rivaroxaban vs warfarin in high risk APS patients with triple aPL-positivity. The primary cumulative outcome measure will be incident acute thrombosis (arterial or venous) confirmed by appropriate imaging studies, major bleeding, or death. Secondary Outcome variables Separate evaluation of arterial and venous thrombosis and all-cause death. The estimated completion date is december 2020.

The largest case series published with APS patients included 35 patients treated with rivaroxaban. Their median follow-up months was 10 . Twenty four of them previously had deep vein thrombosis and 11 patients had additionally experienced pulmonary embolism. Indications for switching from a VKA to rivaroxaban for the secondary prevention of VTE included erratic INR control and INR consistently in the sub-therapeutic range. In the follow-up period, no thrombotic relapse occurred (16). On the other hand, these is another case series report on 8 patients with APS treated with rivaroxaban. Five patients developed arterial events during the treatment with rivaroxaban. All of them had venous thrombosis as the initial manifestation of APS. Author's preliminary experience as well cases previously reported in the literature suggest that there is a high-risk group that is less protected with rivaroxaban, namely those with previous arterial thrombosis or triple positivity (17).

\section{Apixaban for Antiphospholipid Syndrome}

The largest prospective study to date comparing a direct oral anticoagulant with warfarin is Apixaban 
for the Secondary Prevention of Thrombosis Among Patients With Antiphospholipid Syndrome (ASTRO-APS). The patients are being randomized to either adjusted-dose warfarin or apixaban $2.5 \mathrm{mg}$ twice a day. The primary outcomes are the rate of arterial and/or venous thrombosis and major and non-major bleeding. All clinical outcomes will be adjudicated by a panel blinded to the treatment arm (18). Th estimated primary completion date will be december 2019.

There are very few case reports with APS patients treated with apixaban. Despite a similar half-life, pharmacokinetics and pharmacodynamics between apixaban and rivaroxaban, there may be a difference in clinical response in potent hypercoagulable disorders, such APS. One of them raised an important dosing issue between rivaroxaban and apixaban. (19). New medical and pharmacological data are awaited.

\section{Dabigatran for Antiphospholipid Syndrome}

Noel et al published in 2015 a descriptive analysis of 26 patients with APS enrolled in a French multicentre observational cohort receiving DOAC. The main outcomes were the occurrence of a thrombotic recurrence or bleeding events. DOAC were introduced as second-line therapy because of INR lability/therapeutic simplification $(n=17)$, recurrent thrombosis $(\mathrm{n}=1)$, VKA's associated bleeding event $(n=1)$, and atrial fibrillation $(n=1)$. Eleven patients were treated with dabigatran and 15 patients with rivaroxaban. After a median (IQR) follow-up of 19 (829) months, one relapse of arterial thrombosis, two bleeding events (hypermenorrhea and rectal bleeding under rivaroxaban) and one recurrent migraine were reported, leading to discontinuation of therapy in these 4 patients (20).

Another recently published series case report was a retrospective cohort analysis sought to identify patients with APS who have been treated with DOACs. Within 41 patients with confirmed APS, 5 patients were treated with dabigatran, 7 with apixaban and 29 with rivaroxaban. Five patients had recurrent TED events. Two patients (5\%) experienced major bleeding and 5 (12\%) minor bleeding. Fourteen patients (34\%) started on DOACs as the first line regimen while the rest had been previously treated with another anticoagulant. Reasons for switching to DOAC were mostly because of difficulty managing INR's with warfarin in 11 patients $(27 \%)$. Thirty-two pa- tients $(78 \%)$ are still on a DOAC. For these 32 patients, safe and effective anticoagulation with a DOAC was noted for a median of 48 months (range 14-62 months). The rates of recurrent TED and bleeding complications were shown to be similar to previously published studies using warfarin in APS (21).

A new prospective case series of 56 consecutive white Polish patients with APS was published. The patients were switched from VKAs (warfarin or acenocoumarol) to rivaroxaban, dabigatran or apixaban or DOACs during the acute VTE episode and maintained based on the patient preferences. Forty-nine $(87.5 \%)$ patients were treated with rivaroxaban, $4(7.3 \%)$ with dabigatran and $3(5.4 \%)$ with apixaban. The duration of treatment with the DOACs ranged between 2 and 43 months (mean $22.1 \pm 7.8$ months). Six patients $(10.7 \%$, 5.8 per 100 patient-years) experienced recurrent thrombosis, including deep vein thrombosis $(n=4$, including 2 episodes preceded by nonadherence), superficial vein thrombosis $(n=1)$ and non-ST elevation myocardial infarction $(n=1)$. Four out of these 6 patients had triple positive APS. The recurrence rate of VTE on DOACs was 5.8 per 100 patient-years. Two patients $(3.6 \%)$ experienced severe bleeding. The $6 \%$ risk of recurrent VTE on DOACs cannot be neglected and caution is recommended in APS patients with triple positivity (22).

Since published case series reports and studies suggest that dabigatran could be used in patients with APS, larger clinical trials are needed to confirm safety and efficacy.

\section{Adverse events and Direct Oral Anticoagulant's (DOAC's) risks}

All anticoagulants increase bleeding risk. The agents discussed herein generally do not appear to increase bleeding risk more than heparins or vitamin $\mathrm{K}$ antagonists. A specific antidote against dabigatran, idarucizumab (Praxbind) is available. The lack of a specific antidote for the direct factor Xa inhibitors is a concern; however, their half-lives are short, and reversal agents are in development. Dabigatran and rivaroxaban carry the highest of bleeding complications due to a high degree of renal excretion, whereas the risk for apixaban, edoxaban and betrixaban seems lower. Rivaroxaban is not recommended for in individuals with a creatinine clearance $<30 \mathrm{~mL} /$ minute and should not be used in individuals with a 
creatinine clearance $<15 \mathrm{~mL} /$ minute, as well as in those with significant hepatic impairment (ChildPugh Class B and C with coagulopathy) (23).

Dabigatran may be associated with a slightly lower rate of intracranial hemorrhage and death, and a slightly higher risk of gastrointestinal bleeding at the $150 \mathrm{mg}$ twice daily dose (but not $110 \mathrm{mg}$ twice daily) $(24,25)$. Several cases of intracerebral bleeding associated with rivaroxaban have been reported in literature from post-marketing daily practice (26). Apixaban was not associated with gastrointestinal bleeding as already highlighted by the ARISTOTLE trial, and by the meta-analysis performed by Holster et al., indicating apixaban as a possible first line treatment in patients with risk factors for gastrointestinal haemorrhage (26).

In the RE-LY trial, which randomized 18,113 individuals with AF to dabigatran or warfarin, non-bleeding gastrointestinal events (eg, dyspepsia, dysmotility, gastrointestinal reflux) were twice as common in those who received dabigatran. This may limit dabigatran use in some patients(27). Rivaroxaban was also associated with induced liver injury and hepatic failure. The incidence of this complication is still unknown (26).

Artang et al. showed no difference in the rate of myocardial infarction among warfarin versus factor $\mathrm{Xa}$ inhibitors users(28). Dabigatran has some evidence of cardiovascular risk, not supporting potential increased myocardial infarction risk with all DOAC (26). Statistical analysis highlighted a positive association with angina pectoris for both rivaroxaban and dabigatran.

Regarding thrombotic events, strokes and thromboembolic events resulted statistically significant for all DOACs compared to warfarin (26). Now it is well known that Dabigatran has a Boxed Warning regarding the risk of thrombotic events following premature discontinuation.

\section{Vitamin K Antagonists (VKAs) versus Direct Oral Anticoagulants (DOACs), advantages and disadvantages}

The DOACs differ significantly from VKAs in their onset of action, half-life, drug-drug interactions, need for monitoring, ability to monitor, as well as availability of antidotes in the case of excessive bleeding. In some cases, these differences may translate into similar efficacy with easier administration and lower bleeding risk. However, the efficacy and bleeding risk depend on patient variables such as compliance and interacting medications, and all decisions must be individualized to take these factors into account.

Overall, all-cause mortality from DOACs appears to be lower than that from warfarin, driven primarily by a decrease in fatal intracranial bleeding risks (29).

Heparin and VKAs have a relatively narrow therapeutic window and more variable dose-response relationship that depends on a variety of factors requiring for frequent monitoring of clotting times to optimize the therapeutic dose range and prevent bleeding. Dose may be affected by differing bioavailability, diet, and acute medical illnesses. In contrast, the DOACs are generally used without a requirement for monitoring of drug levels or coagulation. This may be an advantage for patients for whom frequent monitoring is a problem.

VKAs pharmacokinetics is dependent on vitamin $\mathrm{K}$ intake, production in the gastrointestinal tract and interaction with hepatic cytochromes. Patients with difficulty controlling the INR benefit from a DOAC because these agents have less variability in drug effect for a given dose than vitamin $\mathrm{K}$ antagonists.

There are several settings in which VKAs is prefered to one of the DOACs, or in which a DOAC is contraindicated. For example, in patients with renal insufficiency. Direct thrombin inhibitors and direct factor Xa inhibitors are renally excreted to variable degrees. Also, DOAC are no used in patients with prosthetic heart valves and pregnancy.

Use of DOACs may be challenging in patients who are unable to take their medication as prescribed. The lack of routine monitoring and short half-lives of these agents make it more difficult to determine if a patient is taking them appropriately. In addition, missing one or two doses can leave the patient inadequately anticoagulated. In contrast, missing a couple of doses of VKAs is unlikely to substantially increase the time outside the therapeutic range.

Regarding the anticoagulation cost in primary APS, there is new data published showing that switching from VKAs to rivaroxaban may be $48 \%$ more expensive for 1 year(30). The number of clinic visits, human costs, time in therapeutic range and warfarin consumption for each patient were considered. Unless ongoing trials demonstrate improved long-term outcomes for rivaroxaban over warfarin, a $69 \%$ time in therapeutic range does not warrant a change to rivaroxaban at a $8 \%$ increased cost (30). 


\section{CONCLUSION}

The use of DOAC agents may represent a major step forward in the treatment of APS. Unlike VKAs, they have few reported drug interactions and they do not interact with food or alcohol intake, thereby resulting in more stable anticoagulant intensity. Most importantly, monitoring their anticoagulant intensity is not routinely required due to their predictable anticoagulant effects.

The $15^{\text {th }}$ International Congress on Antiphospholipid Antibodies Task Force on APS Treatment Trends published in 2017 concluded that there is insufficient evidence to make recommendations regarding DOAC use in APS. It is not known whether the DOACs may be as effective as VKAs, less effec-

\section{REFERENCES}

1. Hochberg M., Silman A., Smoen J., Weinblatt M., Weisman M. Rheumatology. 2015.

2. Ansell J., Hirsh J., Hylek E., Jacobson A., Crowther M., Palareti G. Pharmacology and management of the vitamin $\mathrm{K}$ antagonists: American College of Chest Physicians Evidence-Based Clinical Practice Guidelines (8th Edition). Chest 2008; 133:160S-198S.

3. Ansell J., Hirsh J., Poller L., Bussey H., Jacobson A., Hylek E. The pharmacology and management of the vitamin $\mathrm{K}$ antagonists: the Seventh ACCP Conference on Antithrombotic and Thrombolytic Therapy. Chest 2004;126:204S-233S.

4. Hisada R., Kato M., Sugawara E., Fujieda Y., Oku K., Bohgaki T. et al. Thrombotic risk stratification by platelet count in patients with antiphospholipid antibodies: a longitudinal study. J Thromb Haemost 2017;15:1782-7.

5. Rieder M.J., Reiner A.P., Gage B.F., Nickerson D.A., Eby C.S., McLeod H.L. et al. Effect of VKORC1 haplotypes on transcriptional regulation and warfarin dose. N Engl J Med 2005; 352:2285-93.

6. Schwarz U.I., Ritchie M.D., Bradford Y., Li C., Dudek S.M., FryeAnderson A., et al. Genetic determinants of response to warfarin during initial anticoagulation. N Engl J Med 2008; 358:999-1008.

7. Bodin L., Verstuyft C., Tregouet D-A., Robert A., Dubert L., Funck-Brentano C. et al. Cytochrome P450 2C9 (CYP2C9) and vitamin $\mathrm{K}$ epoxide reductase (VKORC1) genotypes as determinants of acenocoumarol sensitivity. Blood 2005; 106:135-40.

8. Caldwell M.D., Awad T., Johnson J.A., Gage B.F., Falkowski M., Gardina P. et al. CYP4F2 genetic variant alters required warfarin dose. Blood 2008;111:4106-12.

9. Pérez-Andreu V., Roldán V., Antón A.I., García-Barberá N., Corral J., Vicente V. et al. Pharmacogenetic relevance of CYP4F2 V433M polymorphism on acenocoumarol therapy. Blood 2009; 113:4977-9.

10. Rivaroxaban for the treatment of deep vein thrombosis and prevention of recurrent deep vein thrombosis and pulmonary embolism | Guidance and guidelines | NICE n.d.

11. EINSTEIN Investigators, Bauersachs R., Berkowitz S.D., Brenner B., Buller H.R., Decousus H. et al. Oral Rivaroxaban for Symptomatic Venous Thromboembolism. N Engl J Med 2010; 363:2499-510.

12. Schulman S., Kearon C., Kakkar A.K., Mismetti P., Schellong S., Eriksson H. et al. Dabigatran versus Warfarin in the Treatment of Acute Venous Thromboembolism. N Engl J Med 2009; 361:2342-52.

13. Agnelli G., Buller H.R., Cohen A., Curto M., Gallus A.S., Johnson M. et al. Oral Apixaban for the Treatment of Acute Venous Thromboembolism. N Engl J Med 2013;369:799-808. tive or more effective. What is for sure is that in patients treated with DOACs, recurrent clots may occur. On the other hand, some patients with APS develop new clots in spite of being on VKAs in therapeutic range of INR.

However, if a patient has indications for DOAC taking into account the posibilites discussed above, a DOAC with a more steady drug level may be choosed. A twice daily dosed anticoagulant, apixaban (Eliquis) or dabigatra (Pradaxa) is the best choice, as a recent publication suggests, due to a possible more effective anticoagulantion (19). Thus, advantages and disadvantages of each agent must be individualized to the patient and clinical setting.

Conflict of interest: none declared Financial support: none declared

14. Sciascia S., Lopez-Pedrera C., Cecchi I., Pecoraro C., Roccatello D., Cuadrado M.J. Non-vitamin K antagonist oral anticoagulants and antiphospholipid syndrome. Rheumatology (Oxford) 2016; 55:1726-35.

15. Cohen H., Hunt B.J., Efthymiou M., Arachchillage D.R.J., Mackie I.J., Clawson S. et al. Rivaroxaban versus warfarin to treat patients with thrombotic antiphospholipid syndrome, with or without systemic lupus erythematosus (RAPS): a randomised, controlled, open-label, phase 2/3, non-inferiority trial. Lancet Haematol 2016; 3:e426-36

16. Sciascia S., Breen K., BLOOD BH-, 2015 undefined. Rivaroxaban use in patients with antiphospholipid syndrome and previous venous thromboembolism ( $\mathrm{vol} 26, \mathrm{pg} 476,2015)$. LIPPINCOTT WILLIAMS \& WILKINS ... n.d.

17. Signorelli F., Nogueira F., Domingues V., Mariz H.A., Levy R.A. Thrombotic events in patients with antiphospholipid syndrome treated with rivaroxaban: a series of eight cases. Clin Rheumatol 2016; 35:801-5.

18. Woller S.C., Stevens S.M.., Kaplan D.A., Branch D.W., Aston V.T., Wilson E.L. et al. Apixaban for the Secondary Prevention of Thrombosis Among Patients With Antiphospholipid Syndrome. Clin Appl Thromb 2016;22:239-47.

19. Schofield J.R., Hassell K. Dosing considerations in the use of the direct oral anticoagulants in the antiphospholipid syndrome. J Clin Pharm Ther 2018; 43:104-6.

20. Noel N., Dutasta F., Costedoat-Chalumeau N., Bienvenu B., Mariette X., Geffray L. et al. Safety and efficacy of oral direct inhibitors of thrombin and factor $\mathrm{Xa}$ in antiphospholipid syndrome. Autoimmun Rev 2015; 14:680-5.

21. Leblebjian H., Parnes A. Direct Oral Anticoagulants in AntiPhospholipid Syndrome. Blood 2017; 130.

22. Malec K., Góralczyk T., Undas A. The use of direct oral anticoagulants in 56 patients with antiphospholipid syndrome. Thromb Res 2017; 152:93-7.

23. Poulsen B.K., Grove E.L., Husted S.E. New oral anticoagulants: a review of the literature with particular emphasis on patients with impaired renal function. Drugs 2012; 72:1739-53.

24. Desai J., Kolb J.M., Weitz J.I., Aisenberg J. Gastrointestinal bleeding with the new oral anticoagulants - defining the issues and the management strategies. Thromb Haemost 2013; 110:205-12.

25. Southworth M.R., Reichman M.E., Unger E.F. Dabigatran and postmarketing reports of bleeding. N Engl J Med 2013; 368:1272-4.

26. Monaco L., Biagi C., Conti V., Melis M., Donati M., Venegoni M. et al. Safety profile of the direct oral anticoagulants: an analysis of 
the WHO database of adverse drug reactions. Br J Clin Pharmacol 2017;83:1532-43.

27. Bytzer P., Connolly S.J., Yang S., Ezekowitz M., Formella S., Reilly P.A. et al. Analysis of upper gastrointestinal adverse events among patients given dabigatran in the RE-LY trial. Clin Gastroenterol Hepatol 2013; 11:246-52-5.

28. Artang R., Rome E., Nielsen J.D., Vidaillet H.J. Meta-Analysis of Randomized Controlled Trials on Risk of Myocardial Infarction from the Use of Oral Direct Thrombin Inhibitors. Am J Cardiol 2013; 112:1973-9.
29. Chai-Adisaksopha C., Hillis C., Isayama T., Lim W., Iorio A., Crowther M. Mortality outcomes in patients receiving direct oral anticoagulants: a systematic review and meta-analysis of randomized controlled trials. J Thromb Haemost 2015; 13:2012-20.

30. Ciampa A., Salapete C., Vivolo S., Ames P.R.J. Oral anticoagulation cost in primary antiphospholipid syndrome. Blood Coagul Fibrinolysis 2017:1. 